\title{
EFICIENCIA DE LA RESINA LEWATIT MONOPLUS M 600 EN LA REMOCIÓN DE NITRATOS EN AGUAS PARA CONSUMO HUMANO
}

\author{
Tania E. Guerrero Vejarano ${ }^{a^{*}}$, Óscar G. Anaya Fernández
}

\begin{abstract}
RESUMEN
En varias partes del mundo las fuentes de agua son contaminadas por nitratos, principalmente por actividades antropogénicas, siendo una potencial amenaza para la salud, la presente investigación tuvo como objetivo determinar la eficiencia de la resina convencional de intercambio iónico LEWATIT MonoPlus M 600 (aniónica de base fuerte) en la remoción de nitratos, se utilizó el método de espectroscopia Uv-Vis para la cuantificación de nitratos, asimismo se construyó las columnas de intercambio iónico con diámetro de 1", 0,05 L de volumen de resina y una altura de columna de $40 \mathrm{~cm}$, donde se evaluó la eficiencia de las columnas de intercambio iónico, se manejó dos variables, el tiempo de operación y diferentes concentraciones de nitratos. Se determinó que la mayor eficiencia de la resina fue de 99,55 $\%$, a una concentración de 6,45 meq de $\mathrm{NO}_{3-}(400 \mathrm{ppm})$ en un tiempo de $30 \mathrm{~min}$. La eficiencia más baja de 18,61 \% se encontró para la concentración de 16,13 meq de NO3-(1000ppm) en 120 min de operación de la columna. En el análisis de las aguas de Tingo María se determinó que el Pozo 5 de Villa Potokar fue el único que supera los límites máximos permisibles tal como lo indican los estándares de calidad ambiental nacional de agua (10 ppm de $\mathrm{NO}_{3}$ de $\mathrm{N}$ total o $45 \mathrm{ppm} \mathrm{de} \mathrm{NO}_{3}$ ) con una concentración de 52,76 ppm, por lo tanto, no es apta para consumo humano sin previo tratamiento.
\end{abstract}

Palabras clave: Nitratos, resinas de intercambio iónico, eficiencia

\section{EFFICIENCY OF THE LEWATIT MONOPLUS M 600 RESIN IN THE REMOVAL OF WATER NITRATES FOR HUMAN CONSUMPTION}

\begin{abstract}
In several parts of the world the water sources are contaminated by nitrates, mainly by anthropogenic activities, being a potential threat to health, the present investigation had as objective to determine the efficiency of the conventional ion exchange resin LEWATIT MonoPlus M 600 (anionic strong base) in nitrate removal, the Uv-Vis spectroscopy method was used for the quantification of nitrates, as well as the ion exchange columns with a diameter of 1", $0.05 \mathrm{~L}$ of resin volume and a height of $40 \mathrm{~cm}$ column, where the efficiency

\footnotetext{
${ }^{a}$ Universidad Nacional Agraria de la Selva -Carretera Centra km 1.21 Tingo María. Cp 10131 Perú. taniaguerrero@unas.edu.pe

${ }^{\mathrm{b}}$ Ministerio de Vivienda
} 
of the ion exchange columns was evaluated, two variables were managed, the operation time and different concentrations of nitrates. It was determined that the highest efficiency of the resin was $99.55 \%$, at a concentration of $6.45 \mathrm{meq}$ of $\mathrm{NO}_{3-}(400 \mathrm{ppm})$ in a time of $30 \mathrm{~min}$. The lowest efficiency of $18.61 \%$ was found for the concentration of 16.13 meq of NO3$(1000 \mathrm{ppm})$ in $120 \mathrm{~min}$ of column operation. In the analysis of the waters of Tingo María it was determined that Well 5 of Villa Potokar was the only one that exceeds the maximum permissible limits as indicated by the national environmental water quality standards (10 ppm of $\mathrm{NO}_{3}$ of total $\mathrm{N}$ or $45 \mathrm{ppm}$ of $\mathrm{NO}_{3}$ ) with a concentration of $52.76 \mathrm{ppm}$, therefore it is not suitable for human consumption without previous treatment

Key words: Nitrates, ion exchange resins, efficiency

\section{INTRODUCCIÓN}

Generalmente, las fuentes de aguas subterráneas y superficiales son contaminadas, en algunos lugares el agua subterránea sirve como la única fuente de agua potable en las comunidades rurales y las zonas urbanas. Las actividades antropogénicas como la agricultura, la explosión demográfica y la industria generan contaminantes tóxicos tales como aniones inorgánicos, iones metálicos, productos químicos orgánicos sintéticos, residuos orgánicos, etc., que cada año se incrementa sustancialmente que afecta directamente a la calidad de las fuentes de agua. Muchos aniones inorgánicos son tóxicos y nocivos para los seres humanos, principalmente los que no se perciben a simple vista en el agua potable debido a la gran solubilidad que pueden tener en el agua, esto aumenta los posibles riesgos para la salud ${ }^{1}$. Dentro de lo aniones inorgánicos peligroso para los seres vivos están los nitratos $\left(\mathrm{NO}_{3}\right)$. El aumento de los niveles de $\mathrm{NO}_{3-}$ se asocia especialmente al uso intensivo de fertilizantes en la agricultura, lo que ha llevado a una mayor contaminación de las fuentes de agua subterránea y superficial ${ }^{2}$. Los nitratos no se unen fácilmente al suelo, por lo que se lixivian fácilmente, la contaminación del agua ocurre por escorrentía agrícola y urbana, eliminación de aguas residuales no tratadas, desperdicios industriales, fugas de pozos sépticos, lixiviados de vertederos, estiércol animal, desechos de extracción de aire $\mathrm{NO}_{\mathrm{x}}$ de los dispositivos de control de la contaminación del aire $^{3}$. Estos aniones son una gran amenaza para la salud de animales y seres humanos. En seres humanos causa dos efectos para la salud: inducción del "síndrome del bebé azul" (metahemoglobinemia), especialmente en lactantes y formación potencial de nitrosaminas cancerígenas ${ }^{4}$. Los nitratos, debido a su alta solubilidad en agua ${ }^{5}$, un contaminante difícil de eliminarlo en las fuentes de agua en el mundo, en algunos lugares la fuentes de agua tienen capacidad natural de eliminarlos como algunas cuencas donde hay una interface agua dulce y salada, las reacciones de oxidación y reducción pueden atenuar estos contaminantes ${ }^{6}$, pero por lo general es una preocupación mundial, existen varios reportes de investigaciones para eliminar aniones inorgánicos como los $\mathrm{NO}_{3}$, dentro de ellos tenemos el carbón activado que muestra poca adsorción hacia los aniones inorgánicos como los $\mathrm{NO}_{3-}^{7}$, las arcillas capturan cationes y aniones ya sea a través de iones de intercambio, adsorción o ambos sobre todo tienen acción cuando son tratadas con surfactantes ${ }^{8}$, así como también los hidróxidos dobles en capas (LDH), remueven materiales inorgánicos con propiedades deseables para eliminar 
los contaminantes aniónicos del agua como los $\mathrm{NO}_{3-}$, con una eliminación era de $85,5 \%{ }^{9}$, la zeolita cubierta con una capa de quitosano protonada con ácido fue probada satisfactoriamente para eliminar $\mathrm{NO}_{3-}{ }^{10}$. El uso de materiales de desecho agrícola ha sido estudiado para la eliminación de $\mathrm{NO}_{3-}$ a partir de soluciones acuosas ${ }^{11}$ y las resinas de intercambio iónico Purolite A 520 son muy eficientes en la remoción de nitratos ${ }^{10}$. Generalmente, hay varios tipos de resinas de intercambio iónico que se ofertan en el mercado, sin embargo, no todas presentan la misma eficiencia, dicha información es importante para la elegir la resina adecuada, ya que son procesos costosos, en este trabajo se presentan los resultados de la resina convencional aniónica de base fuerte con resultados prometedores a un costo mucho más bajo en donde el objetivo principal fue determinar la eficiencia de la resina antes mencionada.

\section{PARTE EXPERIMENTAL}

1.1 Lugar de ejecución: Se realizó en la Universidad Nacional Agraria de la Selva, Facultad de Recursos Naturales Renovables, Laboratorio de Fitoquímica y Tratamiento de Aguas Residuales.

1.2 Equipos: Bomba peristáltica, espectrofotómetro AQUAMATE ORION 8000 UVvisible, cronómetro. Materiales: material de uso común de laboratorio, tubería de PVC categoría 10 de 1", tapas rosca para tubería de PVC, mangueras, recipiente de plástico de 16 litros. Reactivos: Resina de intercambio iónico LEWATIT MonoPlus M 600 (aniónica de base fuerte), Solución de cloruro de sodio ACS; ISO; Reag. Ph Eur adquirido de Merck, nitrato de potasio $\left(\mathrm{KNO}_{3}\right)$ ACS, ISO, Reag. Ph Eur. Adquirido de Merck

1.3 Diseño y construcción de la columna: Para el diseño y construcción de la columna se tomaron las siguientes consideraciones ${ }^{12,13}$.

Volumen de Servicio: (Vs)

$$
\mathrm{Vs}=\frac{\mathrm{REC}}{\mathrm{IRC}}
$$

Donde: Vs: Volumen de servicio (L/m3), volumen de agua en litros que puede soportar un metro cúbico de resina hasta su regeneración. REC: Capacidad de la resina $\left(\mathrm{kg} / \mathrm{m}^{3}\right)$. IRC: Concentración del ion a eliminar $(\mathrm{kg} / \mathrm{L})$

Tiempo de servicio: $(\mathrm{St})$

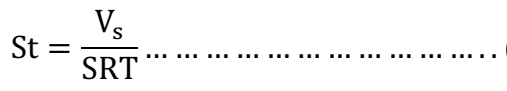

Donde: St: Tiempo de servicio. SRT: Caudal de flujo de servicio $=\mathrm{L} / \mathrm{h} / \mathrm{m}^{3}$

Determinación del volumen de resina: ( $\mathrm{Vol})$

$$
\mathrm{Vol}=\left\lfloor\frac{\mathrm{IRC} * \mathrm{Q}}{\mathrm{REC}}\right\rfloor *[\mathrm{St}]
$$


Donde: Vol: Volumen de resina (L). Q: Flujo total L/h $(5,8 \mathrm{~L} / \mathrm{h})$

Determinación del volumen de la columna: (Vc)

$$
\mathbf{V}_{\mathbf{c}}=\mathrm{Vol}^{*} \mathrm{FE}
$$

Donde: $\mathrm{FE}=4$ (para resinas aniónicas de base fuerte).

Determinación de la altura de la columna

$$
\mathbf{h c}=\frac{\mathrm{Vc} * 4}{\pi * \mathrm{~d}}
$$

Donde:

$\mathbf{V c}=$ volumen de columna (L). $\mathbf{d}=$ diámetro de la tubería en la columna a utilizar (1")

$\mathbf{h}=$ altura de la columna $(\mathrm{cm})$

1.4 Construcción de la curva de calibración para medir la concentración de nitratos: Se pesó $0,18452 \mathrm{~g} \mathrm{KNO}_{3}$, se aforo a un volumen de 250, se tomó $25 \mathrm{~mL}$ de la solución madre y se aforo a $250 \mathrm{~mL}$, a partir de esta solución intermedia se preparó 5 estándares de $0 ; 0,90$; 2,$71 ; 4,52 ; 6,33$ ppm, Los mismos que fueron tratados con $1 \mathrm{~mL}$ de $\mathrm{HCl} 0,1 \mathrm{~N}$, se dejó reposar 30 minutos antes de aforarlos a $50 \mathrm{~mL}$. Se realizó las lecturas en el espectrofotómetro utilizando las cubetas de cuarzo a una longitud de onda de $220 \mathrm{~nm}$.

1.5 Colección de las muestras de agua de Tingo María: Las muestras se tomaron el 16/08/2013 en dos pozos de Castillo Grande, el 04/11/2013 en Brisas del Huallaga, y Villa Potokar, 12/11/2013 en la quebrada Cocheros y Naranjal. Para las muestras de agua de pozo se utilizó el muestreo simple o de sondeo a 4,5 metros de profundidad y para aguas superficiales el muestreo integrado, en ambos casos, fue manual y se siguió la cadena de vigilancia (etiquetado, sellado y registro), las muestras se almacenaron en botellas de vidrio hasta ser analizadas en el laboratorio el mismo día, se tomaron tres muestras por punto de muestreo.

1.6 Determinación de la eficiencia de la columna de resinas de intercambio iónico: La eficiencia de la columna se evaluó a un caudal establecido, se tomó muestras en la salida de la columna, a diferentes tiempos de trabajo tales como: 30, 60, 90 y 120 minutos, en las cuales se determinó la concentración de nitratos a cada muestra recogida y se analizó la concentración de nitratos por triplicado con el espectrofotómetro UV-VIS, la eficiencia se calculó con la siguiente fórmula

$$
\boldsymbol{E} \boldsymbol{f i c i e n c i a}(\%)=\frac{\left[\mathrm{meqNO}_{3}{ }^{-}\right] \text {entrada }-\left[\mathrm{meqNO}_{3}{ }^{-}\right] \text {salida }}{\left[\text { meqNO}_{3}{ }^{-}\right] \text {entrada }} * 100 .
$$

Donde: meq $\mathrm{NO}_{3}$ : miliequivalentes de nitratos

Variables independientes: Tiempo de operación: $30 \mathrm{~min}, 60 \mathrm{~min}, 90 \mathrm{~min}$ y $120 \mathrm{~min}$. Concentración de nitratos: 16,$13 ; 12,9 ; 9,68$ y 6,45 meq.

Variables dependientes: Eficiencia de la remoción de nitratos, disminución de meq $\mathrm{NO}_{3-}$ 
El diseño experimental fue DCA con arreglo factorial de 4x4. El análisis estadístico de los datos se realizó con el software estadístico $\mathbf{R}$

1.7 Análisis de las aguas de Tingo María: Se analizó la concentración de nitratos $\left(\mathrm{NO}_{3}\right)$ de las aguas de consumo de Tingo María, antes y después de ingresar a la columna de intercambio iónico con el método antes descrito dentro de ellas esta: agua subterránea de pozo del centro poblado menor de Castillo Grande, asentamiento humano Brisas del Huallaga, Lotización Villa Potokar y de la ciudad universitaria

\subsection{Determinación de la capacidad real de la resina, volumen de agua de regeneración} y lavado

Determinación de la capacidad real de la resina: Se calculó la capacidad real de la resina, con la cantidad de gramos que ha atrapado ésta durante el proceso hasta su límite. Conociendo el caudal de trabajo y la concentración de las aguas, se procede a realizar los cálculos para determinar la capacidad experimental de la resina

$$
\mathbf{C}=\frac{\mathrm{Tc} * \mathrm{Q} *\left[\mathrm{NO}_{3}{ }^{-}\right]}{\mathrm{Vol}}
$$

Donde: C: capacidad real de la resina (g/L) Tc: Tiempo de ruptura (h); Q: Caudal de entrada del agua a la columna $(\mathrm{L} / \mathrm{h}) ;\left[\mathbf{N O}_{3}{ }^{-}\right]$: Concentración de nitratos $(\mathrm{g} / \mathrm{L})$;

Vol: Volumen de resina (L)

Después de determinar la capacidad de remoción experimental de la resina, se comparó con la capacidad de remoción teórica y se determinó la capacidad real de la resina.

Determinación del volumen de agua de regeneración y lavado: Para regenerar la resina se utilizó una solución de $\mathrm{NaCl}$ al $10 \%$, el volumen se estimó estequimetricamente según el ratio de regeneración $(\mathrm{Rg})$

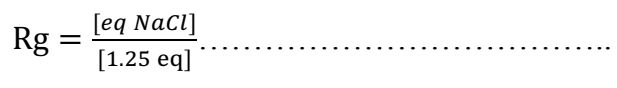

\section{RESULTADOS Y DISCUSIÓN}

\section{Diseño y construcción de la columna:}

Tabla 1. Datos de diseño.

\begin{tabular}{ll}
\hline \multicolumn{1}{c}{ Parámetro de diseño } & \multicolumn{1}{c}{ Cantidad } \\
\hline Volumen de servicio $(\mathrm{Vs})=$ & $77,5 \mathrm{~L} / \mathrm{m} 3$ de resina \\
Tiempo de servicio $(\mathrm{Ts})=$ & $0,66 \mathrm{~h}$ \\
Volumen de columna $(\mathrm{Vc})=$ & $0,1972 \mathrm{~L}$ \\
Volumen de resina $(\mathrm{Vr})=$ & $0,049 \mathrm{~L}$ \\
Altura de columna $(\mathrm{Hc})=$ & $38,919 \mathrm{~cm}$ \\
\hline \hline
\end{tabular}

Fuente: Datos calculados con las ecuaciones de diseño 
En el diseño de las columnas de intercambio iónico se consideró los criterios del Manual de Ingeniero Químico ${ }^{13}$, el diseño coincide con un reporte de columnas hechas a partir de cloruro de polivinilo (PVC) con 1" de diámetro, $16 \mathrm{~cm}$ de longitud con un volumen de 0,06 $\mathrm{mL}$ con resina macroporosa de intercambio catiónico, o LayneRT (Layne Christensen Co., The Woodlands, TX) y una resina de intercambio de aniones modificada con nanopartículas de óxido ferroso ${ }^{12}$, en la presente investigación se usó $0,05 \mathrm{~L}$ de resina a diferencia de lo reportado la cantidad de resina depende de la densidad y las propiedades de las mismas ${ }^{13}$.

\section{Curva de calibración:}

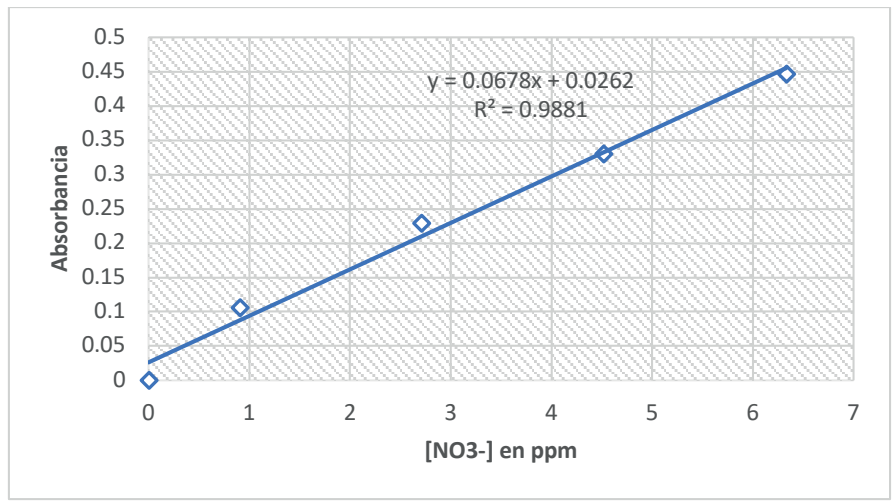

Figura 1. Curva de calibración para determinación de la concentración de nitratos en ppm.

La curva de calibración cumple con la Ley de Beer como se observa en la figura 1, con un coeficiente de correlación $\mathrm{R}^{2}$ óptimo cercano a 1 , por lo tanto, se puede asegurar la confiabilidad de los resultados de las concentraciones de nitratos reportados.

\section{Determinación de la eficiencia de la columna:}

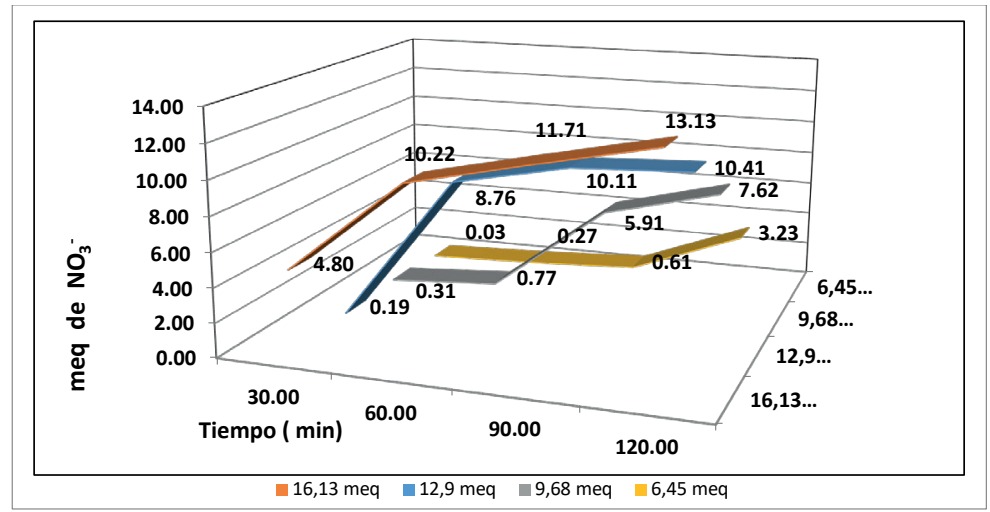

Figura 2. meq. de $\mathrm{NO}_{3}^{-}$remanentes en función del tiempo. 
La cinética de remoción de meq $\mathrm{NO}_{3}^{-}$depende de la concentración inicial de la solución que ingresa a cada columna, el tiempo de operación, el $\mathrm{pH}$ y la capacidad de la resina o absorbente utilizado. La concentración de 6,45 meq de $\mathrm{NO}_{3}^{-}$presentó 0,61 y 3,23 meq de $\mathrm{NO}_{3}^{-}$remanentes a los 90 y 120 minutos, respectivamente, la columna tiene buena capacidad de eliminación hasta los 90 min., luego baja su capacidad en un $50 \%$, aproximadamente. La concentración de 9,68 meq presentó 0,77 meq remanentes a los 60 min, su capacidad de intercambio es óptimo hasta ese tiempo. La concentración de 12,9 meq a los $30 \mathrm{~min}$ presentó 0,19 meq remanentes y la concentración de 16,13 meq de 4,80 meq, se aprecia que la capacidad de intercambio de la primera es óptima hasta los $30 \mathrm{~min}$ y la segunda debido a la alta concentración de meq no realiza el intercambio iónico de manera eficiente (figura 2). Los tiempos ya mencionados alcanzan una considerable eliminación de nitratos, la resina es óptima para remover los nitratos si comparamos con los reportes siguientes. La capacidad de adsorción de la tela de carbono tratada con ácido para remover $\mathrm{NO}_{3}^{-}$y $\mathrm{NO}_{2}^{-}$, fue de 2,03 y $1,01 \mathrm{mmol} / \mathrm{g}$, respectivamente ${ }^{7}$. Entre todas las organoarcillas, HDTMA QLD-bentonita modificada mostraron mejor resultado: H-B-2CEC y H-B-4CEC podrían eliminar 0,20 meq y 0,24 meq de $\mathrm{NO}_{3}-\mathrm{g}$ de organoarcilla, respectivamente ${ }^{8}$. Los hidróxidos dobles preparados (Ni-Fe) fue estudiado su potencial para eliminar $\mathrm{NO}_{3}{ }^{-}$usando agua de mar enriquecida $\mathrm{NO}_{3}{ }_{3}$ a una concentración de $40 \mathrm{~mol} / \mathrm{dm}^{3}$, donde la máxima de $\mathrm{NO}_{3}^{-}$fue de $0,33 \mathrm{mmol} / \mathrm{g}$ cuando se añadió $\mathrm{LDH}(\mathrm{Ni}-\mathrm{Fe})^{9}$. La Chitosan cubierta con zeolita $(\mathrm{Ch}-\mathrm{Z})$ protonado con $\mathrm{HCl}$, tiene capacidad de intercambio iónico para $\mathrm{NO}_{3}^{-}$de $0,74 \mathrm{mmol} \mathrm{NO}_{3}^{-} \mathrm{g}$ de $\mathrm{Ch}-\mathrm{Z}^{10}$. Las resinas y adsorbentes antes mencionados tienen poca capacidad de intercambio iónico a diferencia de la resina de intercambio iónico selectiva para nitrato Purolite A $520 \mathrm{E}^{14}$ que tiene una alta capacidad de intercambio iónico, la idea es dar otras alternativas a la resina especifica considerando los costos y la accesibilidad de la misma sobre todo para zonas rurales y urbanas donde las fuentes de agua, principalmente las subterráneas son contaminadas por nitratos.

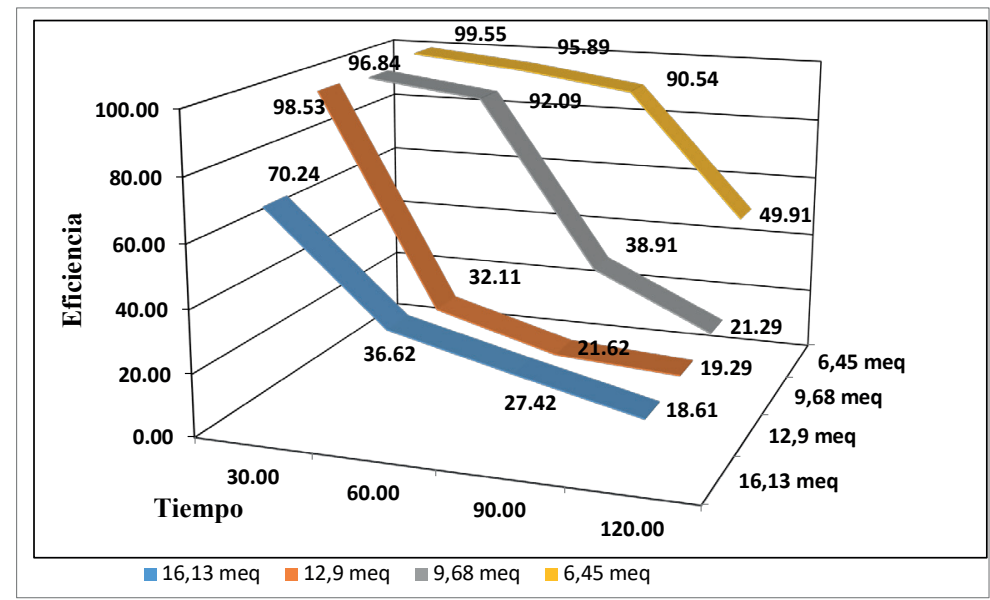

Figura 3. Eficiencia de la columna en función del tiempo de operación. 
Después de analizar los meq de $\mathrm{NO}_{3}^{-}$remanentes en función del tiempo, se evaluó la eficiencia de las columnas de intercambio iónico con la resina antes mencionada y extrapolar datos respecto a que tan eficientes son a diferentes concentraciones y hasta que tiempo de operación trabaja de manera óptima el intercambio iónico, estos datos son importantes para los interesados en remover nitratos que como ya se dijo causan problemas de salud. Se determinó que 6,45 meq de $\mathrm{NO}_{3}{ }^{-}$presentó un buen intercambio iónico hasta los 90 min con una eficiencia de 90,54\%, 9,68 meq de $\mathrm{NO}_{3}^{-}$hasta los 60 min tuvo una eficiencia de 92,09\%, 12,9 meq de $\mathrm{NO}_{3}^{-}$con 98,53\% de eficiencia a los 30 min y finalmente la solución más concentrada que se evaluó de 16,13 meq con una eficiencia de 70,24 \% (figura 2). Uno de los absorbentes de arcilla, montmorrillonita de calcio activada por $\mathrm{HCl}$ mostró hasta una capacidad de eliminación de $\mathrm{NO}_{3}{ }_{3}^{-}$, hasta $22,28 \%{ }^{8}$. La eliminación de $\mathrm{NO}_{3}{ }^{-}$con Hidróxidos de doble capa (LDH) se encontró que era $85,5 \%$ bajo ciertas condiciones, usando 0,3 g de LDH en $100 \mathrm{~mL}$ de solución de $\mathrm{NO}_{3}{ }^{-}$con una concentración inicial de $10 \mathrm{mg} / \mathrm{L}^{9}$. La resina ALRAE, presentó una capacidad de absorción de $87,2 \%$, el proceso fue de aproximadamente 10 $\min ^{15}$. Las columnas de intercambio iónico presentan una buena eficiencia, considerando que se usó concentraciones muy altas con respecto a las concentraciones que normalmente se encuentran las fuentes de agua ya sea subterránea o superficial.

\section{Análisis de las aguas de Tingo María}

Tabla 2. Eficiencia de remoción de nitratos $\left(\mathrm{NO}_{3}^{-}\right)$de las aguas de Tingo María.

\begin{tabular}{|c|c|c|c|c|}
\hline Muestras & Lugar & $\begin{array}{c}\text { Concentración } \\
\text { Entrada } \\
(\text { ppm) }\end{array}$ & $\begin{array}{l}\text { Concentración } \\
\text { Salida (ppm) }\end{array}$ & $\begin{array}{c}\text { Eficiencia } \\
(\%)\end{array}$ \\
\hline 1 & $\begin{array}{l}\text { Quebrada Cocheros } \\
\text { (reservorio) }\end{array}$ & 13,16 & 0,02 & 99,84 \\
\hline 2 & Quebrada Naranjal (reservorio) & 14,38 & 0,04 & 99,70 \\
\hline 3 & Pozo 1 (Castillo Grande) & 18,2 & 0,08 & 99,57 \\
\hline 4 & Pozo 2 (Castillo Grande) & 19,4 & 0,08 & 99,58 \\
\hline 5 & Pozo 3 (Brisas del Huallaga) & 22,81 & 0,15 & 99,34 \\
\hline 6 & Pozo 4 (Villa Potokar) & 43,88 & 0,23 & 99,48 \\
\hline 7 & Pozo 5 (Villa Potokar) & 52,76 & 0,58 & 98,90 \\
\hline
\end{tabular}

Fuente: Datos reportados del Espectrofotómetro Orion Aquamate UV-vis 8000.

Después de analizar la concentración de $\mathrm{NO}_{3}^{-}$en las aguas subterráneas y las quebradas, la mayoría están por debajo de 45 ppm, que es el límite máximo permisible tal como lo indica los estándares de calidad ambiental nacional de agua $\left(10 \mathrm{ppm}\right.$ de $\mathrm{NO}_{3}^{-}$de $\mathrm{N}$ total o 45 ppm de $\mathrm{NO}_{3}$ ), el único que supera los límites máximos permisibles es el Pozo 5 con una concentración de 52,76 ppm. Asimismo, la columna operó eficientemente con un promedio de aproximadamente $99 \%$ de remoción de nitratos en las aguas de Tingo María. 


\section{Determinación de la capacidad de la resina, volumen de agua de regeneración y lavado}

Se obtuvo que la resina atrapa $69,9 \mathrm{~g} / \mathrm{L}$ por lo tanto, la capacidad real de la resina es de 1,112 eq/L, aproximándose al dato teórico del fabricante de 1,25 eq/L. Se determinó que el agua del primer lavado fue aproximadamente $0,5 \mathrm{~L}$ por espacio de $10 \mathrm{~min}$, seguido de una generación con $\mathrm{NaCl}$ al $10 \%$ con un volumen de 1,2 L durante 10 min y finalmente el lavado 2 con 1,5 L de agua durante 15 min.

\section{CONCLUSIONES}

Se determinó que la mayor eficiencia de la resina de intercambio iónico LEWATIT MonoPlus M 600 (aniónica de base fuerte) fue de 99,55 \%, para la concentración de 6,45 meq de $\mathrm{NO}_{3}$. (400 ppm) en un tiempo de 30 min. La eficiencia más baja de 18,61\% se encontró en la concentración de 16,13 meq de $\mathrm{NO}_{3}^{-}(1000 \mathrm{ppm})$ en 120 min de operación de la columna. Asimismo, se puede concluir que la resina antes mencionada presenta una considerable capacidad de intercambio iónico a las siguientes condiciones de concentración de meq de $\mathrm{NO}_{3}^{-}: 12,9$ con una eficiencia de $96,84 \%$ hasta los $30 \mathrm{~min}, 9,68$ con eficiencia de $92,09 \%$ hasta los 60 min y finalmente para 6,45 con una eficiencia de $90,54 \%$ a los 90 min. Estos datos sirven para extrapolar resultados sobre concentraciones de nitratos a evaluar y estimar el tiempo de operación óptima de la columna. Se obtuvo que la capacidad real de la resina es de 1,112 eq/L, aproximándose al dato teórico del fabricante de 1,25 eq/L.

En el análisis de las aguas de Tingo María se determinó que el Pozo 5 de Villa Potokar fue el único que supera los límites máximos permisibles, tal como lo indica los estándares de calidad ambiental nacional de agua (10 ppm de $\mathrm{NO}_{3}$ de $\mathrm{N}$ total o $45 \mathrm{ppm}$ de $\mathrm{NO}_{3}$ ) con una concentración de $52,76 \mathrm{ppm}$, por lo tanto, no es apta para consumo humano sin previo tratamiento.

\section{AGRADECIMIENTOS}

Se agradece al Ing. José Luis Paredes Salazar, por el apoyo sobre información de la resina a utilizar y su apoyo con el Laboratorio de Análisis de Tratamiento de Aguas, de igual manera al técnico del laboratorio Carlos Remigio Salazar Salazar.

\section{REFERENCIAS BIBLIOGRÁFICAS}

1. Velizarov S, Crespo JG, Reis MA. Removal of inorganic anions from drinking water supplies by membrane bio/processes. Rev Environ Sci Bio. 2004; 3: 361-380

2. Rao EVSP, Puttanna K. Nitrates, agriculture and environment. Curr Sci. 2000; 79:11631168

3. Romano N, Zeng C. Evaluating the newly proposed protocol of incorporated potassium in nitrate toxicity experiments at different salinities: a case study with the tiger prawn, Penaeus monodon, juveniles. Aquaculture. 2009; 289:304-309. 
4. Majumdar D, Gupta N. Nitrate pollution of groundwater and associated human health disorders. Ind J Environ Hlth. 2000; 42:28-39.

5. Thomson TS. Nitrate concentration in private rural drinking water supplies in Saskatchewan, Canada. Bull Environ Cont Toxicol. 2001; 66:64-70.

6. Valiente N, Carrey R, Otero N, Soler A, Sanz D, Muñoz-Martín A, et al. A multi-isotopic approach to investigate the influence of land use on nitrate removal in a highly saline lake-aquifer system Albacete, Spain 2018 Science of the Total Environment 631-632 (2018) 649-659

7. Afkhami A, Madrakian T, Karimi Z. The effect of acid treatment of carbón cloth on the adsorption of nitrite and nitrate ions. J Hazard Mater. 2007; 144:427-431.

8. Bhattacharyya KG, Gupta SS. Adsorption of a few heavy metals on natural and modified kaolinite and montmorillonite: a review. Adv Colloid Interf Sci. 2008; 140:114-131.

9. Xi Y, Mallavarapu M, Naidu R. Preparation, characterization of surfactants modified clay minerals and nitrate adsorption. Appl Clay Sci. 2010; 48:92-96.

10. Schick J, Caullet P, Paillaud J, Patarin J, Mangold-Callarec C. Batch-wise nitrate removal from water on a surfactant-modified zeolite. Micro Meso Mater. 2010; 132:395-400.

11. Orlando US, Baes AU, Nishijima W, Okada M. A new procedure to produce lignocellulosic anion exchangers from agricultural waste materials. Bioresour Technol. 2002; 83:195-198.

12. William A, Ileana W, Wiprächtigerc M, Kara L. Effects of operating and design parameters on ion exchange columns for nutrient recovery from urine. Environ Sci Water Res Technol. 2018: 4: 828-838.

13. Perry RH, Green DW. Manual del Ingeniero Químico. 6ta Edición. Madrid: Editorial McGraw-Hill; 2001. 2577 p.

14. Saba S, Nalan K, Ümran Y, Muserref A, Mithat Y. Removal of nitrate from aqueous solution by nitrate selective ion exchange resins. React Funct Polym. 2006; 66:12061214.

15. Xing X, Baoyu G, Yaqing Z, Suhong C, Xin T, Qinyan Y, et al. Nitrate removal from aqueous solution by Arundo donax L. reed based anion exchange resin. J Hazard Mater. 2012; 203-204:86- 92 